\title{
DART: Open-Domain Structured Data Record to Text Generation
}

\author{
Linyong Nan ${ }^{1}$ Dragomir Radev ${ }^{1,2} \quad$ Rui Zhang $^{3} \quad$ Amrit Rau $^{1}$ Abhinand Sivaprasad $^{1}$ \\ Chiachun Hsieh $^{4}$ Xiangru Tang $^{1}$ Aadit Vyas $^{1}$ Neha Verma $^{1} \quad$ Pranav Krishna $^{5}$ \\ Yangxiaokang Liu ${ }^{1} \quad$ Nadia Irwanto $^{1}$ Jessica Pan $^{1} \quad$ Faiaz Rahman $^{1}$ Ahmad Zaidi $^{1}$ \\ Mutethia Mutuma $^{1} \quad$ Yasin Tarabar $^{1} \quad$ Ankit Gupta $^{1} \quad$ Tao Yu$^{1} \quad$ Yi Chern Tan $^{1}$ \\ Xi Victoria Lin ${ }^{2 *}$ Caiming Xiong $^{2} \quad$ Richard Socher $^{2} \quad$ Nazneen Fatema Rajani $^{2}$ \\ ${ }^{1}$ Yale University ${ }^{2}$ Salesforce Research ${ }^{3}$ Penn State University \\ ${ }^{4}$ The University of Hong Kong ${ }^{5}$ MIT \\ \{linyong.nan, dragomir.radev\}@yale.edu, rmz5227@psu.edu, nazneen.rajani@salesforce.com
}

\begin{abstract}
We present DART, an open domain structured DAta-Record-to-Text generation dataset with over 82k instances (DARTs). Data-to-text annotations can be a costly process, especially when dealing with tables which are the major source of structured data and contain nontrivial structures. To this end, we propose a procedure of extracting semantic triples from tables that encodes their structures by exploiting the semantic dependencies among table headers and the table title. Our dataset construction framework effectively merged heterogeneous sources from open domain semantic parsing and spoken dialogue systems by utilizing techniques including tree ontology annotation, question-answer pair to declarative sentence conversion and predicate unification, all with minimum post-editing. We present systematic evaluation on DART as well as new state-of-the-art results on WebNLG 2017 to show that DART (1) poses new challenges to existing data-to-text datasets and (2) facilitates out-of-domain generalization. Our data and code can be found at https: / / github. com/Yale-LILY/dart.
\end{abstract}

\section{Introduction}

Automatically generating textual descriptions from structured data improves the accessibility of knowledge bases to lay users. Such applications include explaining data records to non-experts (Cawsey et al., 1997), writing sports news (Chen and Mooney, 2008), summarizing information in multiple documents (Fan et al., 2019), and generating dialogue responses (Wen et al., 2015).

While significant progress has been made in this field, there are still several issues with existing Data-to-Text datasets. First, they adopt a flat ontology structure of the data, such as slot-value pairs for data records (Lebret et al., 2016; Novikova et al., 2017b) or flat schema for tables (Wiseman et al.,

\footnotetext{
${ }^{*}$ Now at Facebook AI.
}

2017; Chen et al., 2020a; Parikh et al., 2020). This flat structure is not powerful enough to encode rich semantic relationships in the ontology of the structured data, especially tables, whose representation can be further improved with these semantic knowledge. Second, some of the datasets only focus on a small number of domains or knowledge graphs, therefore providing limited number of predicates and data ontologies. For example, E2E (Novikova et al., 2017b) on restaurants and WebNLG (Gardent et al., 2017) on 15 categories from DBPedia. Furthermore, some of them only have loose alignments between data input and sentence due to the nature of the task (Wiseman et al., 2017) and the automatic generation procedure (Vougiouklis et al., 2018; Elsahar et al., 2018).

To address some of these issues and to encourage further research in natural language generation from structured data, we introduce DART, a large and open-domain structured DAta-Record-to-Text generation corpus. The goal of DART is to harvest the diverse predicates occurred in Wikipedia tables, which is significantly richer than those defined in the domain specific ontologies E2E and WebNLG were built on (Table 2). We also introduce a novel tree ontology annotation approach on tables, which converts a flat table schema into a tree structured semantic frame. The tree ontology reflects the core and auxiliary relations in the table schema, and naturally occurs across many domains. As a result, DART provides high-quality sentence annotations to tree structured semantic frames extracted from various data sources, including WikiSQL (Zhong et al., 2017) and WikiTableQuestions (Pasupat and Liang, 2015), two open-domain question answering datasets, as well as E2E (Novikova et al., 2017b) and WebNLG (Gardent et al., 2017) (Figure 1). We evaluated several state-of-the-art data-to-text models on DART, and found that while these models achieve impressive performance on domain-specific datasets, their performance suffers 
on DART due to its open-domain nature and richer semantic structures.

Our contributions are as follows. (1) We present a large and open-domain corpus for structured data record to text generation, annotated with tree ontologies converted from the table. This hierarchical input differentiates our corpus from existing datato-text corpora. (2) We benchmark several stateof-the-art data-to-text models to show that DART introduces new generalization challenges. (3) We demonstrate that using DART for data augmentation improves the performance of existing models on the WebNLG 2017 dataset. We expect the results to generalize to other data-to-text datasets given the open-domain nature of DART.

\section{DART Data Collection}

As shown in Figure 1, DART is constructed from three different sources: (1) human annotation on Wikipedia tables from two table semantic parsing and question answering datasets WikiSQL and WikiTableQuestions (§ 2.1), (2) automatic conversion of questions in WikiSQL to declarative sentences (§ 2.2), and (3) incorporation of existing datasets including WebNLG 2017 and Cleaned E2E (§ 2.3). After collecting the $\langle$ triple-set, sentence $\rangle$ pairs from various data sources, we manually canonicalized the predicates and show that DART covers a broad range of topics ( $\$ 2.4)$. Finally, we discuss the data split in $\S 2.5$.

\subsection{Tree Ontology and Sentence Annotation on Tables}

Tables are a major source of structured data that contain a wealth of information complementary to text and knowledge graphs. We aim to collect $\langle$ triple-set, sentence $\rangle$ pairs from open-domain Wikipedia tables. However, table schema are flat, making them not directly usable for building subject-predicate-object triples to capture rich relationships in the data.

As shown in Figure 2, we propose a two-stage annotation process that involves two groups of annotators: internal annotators and Amazon Mechanical Turk $^{1}$ workers. In the first stage, skilled internal annotators specify the parent of every column header to construct a tree-structured ontology for each table. In the second stage, both internal and external annotators provide a sentential description of the

\footnotetext{
${ }^{1}$ https: / / www . mturk. com/
}

highlighted cells in a row that are automaticallychosen based on the ontology.

Tree Ontology Annotation For each column in a given table, our internal annotators labeled its ontological parent. In Figure 2, for example, the annotator would provide the sequence N NULL, TEAM, Stadium, Stadium, TEAm $\}$ as the parent of each column - column TEAM has no parent, STADIUM has parent TEAM, and so on. In many cases, the relationship between a parent column and its child column can be conceptualized as a "has-a" relationship. For tables that are malformed or have duplicate or missing column names (as shown in Figure 5 of the Appendix), annotators either changed or added appropriate column names in order to fit these patterns. For each table we generate an ontology tree whose root is always [TABLECONTEXT]. This root node either has (1) one child node [TITLE] in the cases where the table title is the subject of entire table, or (2) column header node(s) and a [TITLE] node as children, as shown in Figure 2. This is because in some tables, the table title itself is more appropriate to be the root of the ontology tree (example shown in Figure 6 of the Appendix). In these cases, annotators assigned the special token [TITLE] as the parent of the relevant column nodes. For other tables, title usually provides important context for understanding the table's rows (example shown in Figure 7 of the Appendix). In such cases, [TITLE] is made a child of [TABLECONTEXT] together with the column headers that are appropriate.

We evaluate the quality of the initial tree ontology annotation and made corrections with the following procedure: (1) reject and request corrections from the original annotators if the provided ontology is disconnected or contains a cycle, (2) verify that all column headers appear as a node in the tree. For many tables, the determination of an ontology is a subjective process with many "correct" answers - for example, swapping the positions of TEAM and CITY in the tree in Figure 2 produces an equally valid ontology for the referenced table. If there are multiple ways to construct an ontology based on annotators' decisions of attribute relationships among column headers, we manually unify the annotations for similar tables (for examples, tables about athletes in different sports). The ontologies exhibit a great deal of structural variety. Relevant statistics are summarized in Table 7 and Figure 3 of the Appendix. 


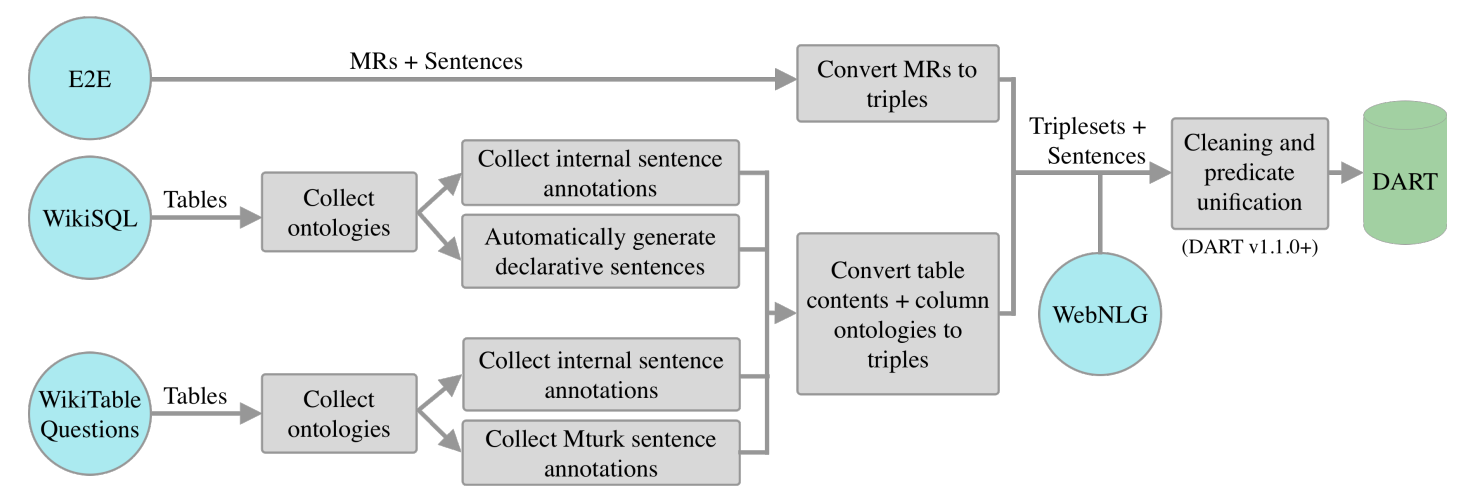

Figure 1: DART data collection pipeline. MR: Meaning Representation.

\begin{tabular}{lcccccc}
\hline & Input Unit & Examples & Vocab Size & Words per SR & Sents per SR & Tables \\
\hline WikiTableText & Row & 13,318 & - & 13.9 & 1.0 & 4,962 \\
LogicNLG & Table & 37,015 & $122 \mathrm{~K}$ & 13.8 & 1.0 & 7,392 \\
ToTTo & Highlighted Cells & 136,161 & $136 \mathrm{~K}$ & 17.4 & 1.0 & 83,141 \\
DART & Triple Set & 82,191 & $33.2 \mathrm{~K}$ & 21.6 & 1.5 & 5,623 \\
\hline
\end{tabular}

Table 1: DART compared with other open-domain table-to-text datasets. DART takes triple sets as input by incorporating the ontology of table headers and title, and its surface realizations tend to be longer with more than single sentence verbalization. SR: Surface Realization.

\begin{tabular}{|c|c|c|c|c|c|c|}
\hline & \multicolumn{6}{|c|}{ DART: 62,659 train / 6,980 dev / 12,552 test } \\
\hline & \multicolumn{2}{|c|}{ WikiTableQuestions } & \multicolumn{2}{|c|}{ WikiSQL } & \multirow{2}{*}{ WebNLG } & \multirow{2}{*}{ Cleaned E2E } \\
\hline & Internal & MTurk & Internal & Declarative & & \\
\hline Domains & \multicolumn{4}{|c|}{ Wikipedia (open-domain) } & 15 DBPedia Categories & Restaurants \\
\hline Unique Predicates & 1,950 & 1,403 & 493 & 2,008 & 347 & 7 \\
\hline Unique Triples & 13,505 & 5,541 & 1,648 & 7,787 & 3,220 & 946 \\
\hline Tripleset-Sentence Pairs & 4,902 & 2,120 & 772 & 4,204 & 27,731 & 42,462 \\
\hline Triples per Tripleset (min, med, max) & $1,3,10$ & $1,3,7$ & $1,2,7$ & $1,2,10$ & $1,3,7$ & $1,4,7$ \\
\hline Vocab Size & $13.4 \mathrm{~K}$ & $8.9 \mathrm{~K}$ & $3.0 \mathrm{~K}$ & $10.7 \mathrm{~K}$ & $8.0 \mathrm{~K}$ & $3.0 \mathrm{~K}$ \\
\hline Words per SR & 15.2 & 16.5 & 14.0 & 12.6 & 22.5 & 22.9 \\
\hline Sentences per SR & 1.0 & 1.1 & 1.0 & 1.0 & 1.4 & 1.6 \\
\hline
\end{tabular}

Table 2: Statistics of DART decomposed by different collection methods. DART exhibits a great deal of topical variety in terms of the number of unique predicates, the number of unique triples, and the vocabulary size.

Connected Component Extraction After we annotated the ontology, we automatically choose a subset of cells for a selected table row to form the triple set. Randomly selecting cells leads to poor quality annotation as the selected data could lack a subject, lack cohesion, or would require information not encoded in the ontology to form a coherent sentence. For example, in Figure 2, if only two nodes CITY and CAPACITY were highlighted then a coherent sentence cannot be produced as there is no direct logical relationship (functional dependency) between them. To solve these issues, instead of randomly selecting cells in a row, we extract connected components from the ontology.

The extracted components have two controllable properties: size and shape. To create variation in size, we randomly sampled between $[2,5]$. The shape is determined by two numbers: the number of sibling node pairs and parent-child node pairs. Increasing the number of sibling node pairs creates a wider tree, while increasing the latter creates a deeper tree. We created a sliding scale between width and depth using an expansion parameter, $p$. We recursively visit a node if it has children with probability $p$ and otherwise move to a sibling if it exists. If $p=1$, the search becomes a DFS and if $p=0$, it becomes BFS. We found that randomly selecting $p$ from 0.5 to 0.7 created a reasonable variation in extracted component shapes. This ensures the balance between breadth and depth of ontology coverage of the selected cells, therefore ensuring the quality of the sentence annotation.

Sentence Annotation Given the table, title, and connected highlighted cells of a row, annotators 


\begin{tabular}{|c|c|c|c|c|c|}
\hline \multirow{4}{*}{$\begin{array}{l}\text { Parent-child } \\
\text { relations provided } \\
\text { by internal } \\
\text { annotator }\end{array}$} & \multicolumn{5}{|c|}{ [TITLE]: NFL Europe Stadiums } \\
\hline & & Team & Stadium & Stadium & Team \\
\hline & Team & Stadium & Capacity & Opened & City \\
\hline & Amsterdam Admirals & Amsterdam Arena & 51,859 & 1996 & Amsterdam, The Netherlands \\
\hline \multirow{2}{*}{$\begin{array}{l}\text { Surface realization } \\
\text { provided by } \\
\text { internal / MTurk } \\
\text { annotator }\end{array}$} & Amsterdam Admirals & Olympisch Stadion & 31,600 & 1928 & Amsterdam, The Netherlands \\
\hline & Barcelona Dragons & Mini Estadi & 15,276 & 1982 & Barcelona, Spain \\
\hline
\end{tabular}

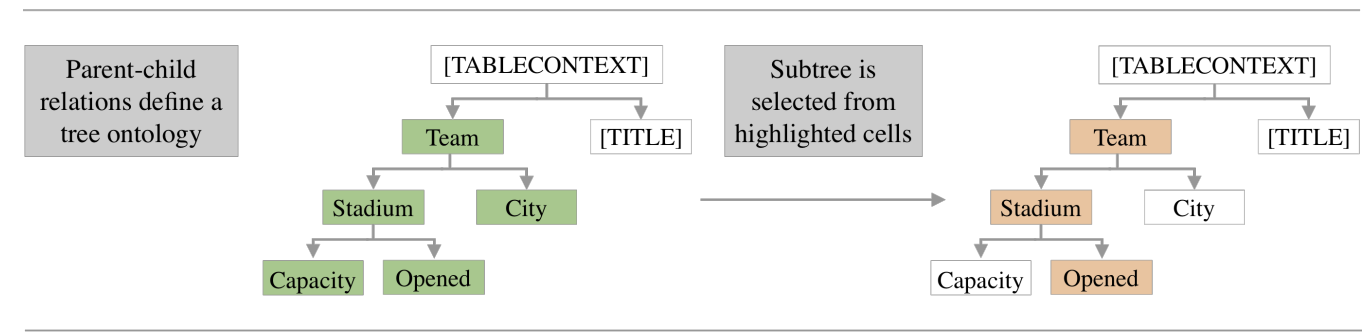

\begin{tabular}{|c|c|c|c|c|}
\hline . & Team & Stadium & Opened & \\
\hline extracted from & $\begin{array}{l}\text { Amsterdam } \\
\text { Admirals }\end{array}$ & $\begin{array}{l}\text { Olympisch } \\
\text { Stadion }\end{array}$ & 1928 & $\begin{array}{l}\text { (Amsterdam Admirals, Stadium, Olympisch Stadion) } \\
\text { (Olympisch Stadion, Opened, 1928) }\end{array}$ \\
\hline
\end{tabular}

Figure 2: Overview of our human annotation procedure. Top panel: We collect the parent-child relations between columns from internal annotators (yellow is parent, green is child). Then, we collect a surface realization of the cells highlighted in orange. Middle panel: We use the provided parent-child relations to construct an ontology tree on the columns, then select the nodes corresponding to the highlighted cells. We gather a connected subtree by collecting all nodes leading up to the highlighted cells' lowest common ancestor. Bottom panel: We extract a set of triples from the subtree as shown. This triple-set is paired with the provided realization to form a DART instance.

were asked to write a description of the highlighted cells. We encouraged the annotators to use diverse vocabulary and syntactic structures. To ensure quality, internal annotators reviewed every crowd sourced sentence for correctness. They either rewrote or discarded the sentences that were nonsensical or incorrect. In some cases, they also changed cell highlighting patterns to match the sentence provided.

Build Tripleset-Sentence Pairs Finally, we convert the highlighted cells to triplesets. For a row $R$, we start with the table's column ontology $T$. We first place the cell values in $R$ in their corresponding slots in $T$, e.g. in Figure 2 we fill TEAM with "Amsterdam Admirals". We then check that the nodes of $T$ corresponding to the highlighted cells in $R$ form a connected subtree. If not, we walk up the tree and highlight each traversed node up until the lowest common ancestor of the highlighted nodes (inclusive) to form a connected subtree. For each node $N$ in the tree except the root node, we can extract the triple (parent $(N)$, title $(N), N)$. For example, since STADIUM is highlighted in Figure 2, we extract the triple (Amsterdam Admirals, STADIUM, Olympisch Stadion). A small number of triple-sets contained more than 10 triples. We discarded these because their associated surface realizations were of poor quality. The numbers of tripleset-sentence pairs annotated by different annotators are shown in Table 2.

\subsection{Automatically Converting Questions to Declarative Sentences}

High quality natural language questions in open domain semantic parsing datasets such as WikiSQL and QA2D techniques found in automatically constructing NLI datasets (Demszky et al., 2018) present themselves as an attractive opportunity to semi-automatically construct an abundance of declarative sentences and align to table cells. We leveraged rule-based QA2D technique ${ }^{2}$ together with manual screening to combine WikiSQL questions and SQL-retrieved-answers into declarative sentences and manually filtered out bad sentences.

We only execute SQL queries without aggregate commands ${ }^{3}$ to retrieve answers corresponding to questions answerable by single rows. An example of such conversion is as follows:

\footnotetext{
${ }^{2}$ We use the rule-based model from https: / / github $\mathrm{com} / \mathrm{kelvinguu/qanli}$ (Demszky et al., 2018). The neural model code is not released.

${ }^{3}$ MAX, MIN, COUNT, SUM, AVG, JOIN, INTERSECT, UNION, GROUP BY, ORDER BY.
} 
Question: In which year did Greece hold its last Summer Olympics?

Answer: 2004

Declarative Sentence: Greece held its last Summer Olympics in 2004.

Alignment with table cells is done at two stages. We first align sentences with corresponding rows by changing SQL commands to SELECT * and use string matching to obtain columns and column headers relevant to the answer and WHERE condition. After manually filtering out bad sentences, bad alignments, or tables without ontology annotations, we were able to get 4,204 sentences. Finally, the corresponding table cells are then converted into triples in the same way as we described in Section 2.1.

Examples of produced declarative sentences can be found in Figure 10 of the Appendix.

\subsection{Incorporating Existing Datasets}

Since they provide a large amount of strictly aligned data-text pairs with high quality sentences, we incorporate the following existing datasets in the same 〈triple-set, sentence〉 pair format with some modifications.

WebNLG 2017 An instance of the WebNLG dataset contains a set of triples extracted from DBpedia and the target text written by human. We include the WebNLG 2017 dataset $^{4}$ consisting of 27731 triple-set sentence pairs with up to $7 \mathrm{RDF}$ triples in a triple set covering 15 domains.

Cleaned E2E The original E2E dataset includes dialogue act meaning representations (MR) and natural language references in the restaurant domain. Later, Dušek et al. (2019) provide Cleaned $\mathrm{E} 2 \mathrm{E}^{5}$ by automatically fixing the dialogue acts to account for omissions and hallucinations in the text. We incorporate Cleaned E2E because of its strict alignment between the meaning representation and the text. To convert the MR to a triple-set, we take the NAME slot (present in almost all the MRs) as the subject. For example, the MR (NAME[ALIMENTUM], AREA[CITY CENTRE], FAMILYFRIENDLY[NO]) is converted to the

\footnotetext{
${ }^{4}$ https://gitlab.com/shimorina/ webnlg-dataset/-/tree/master/webnlg challenge_2017

${ }^{5}$ https://github.com/tuetschek/ e2e-cleaning
}

triple-set \{(ALIMENTUM, AREA, CITY CENTRE), (ALIMENTUM, FAMILYFRIENDLY, NO) \}. We drop MRs which do not contain the NAME slot.

\subsection{Predicate Unification}

We canonicalized the predicates in our triple sets such that those of the same meaning are also represented the same. We manually constructed a predicate mapping table to achieve this. As an example, our predicate mapping maps "Hometown," "Home Town," and "Home Town/City" to the unified predicate "HOMETOWN."

After unifying predicates, we evaluated the diversity of DART by counting the number of unique predicates in its partitions. As shown in Table 2, we see that the Wikipedia partition of DART contains much more unique predicates than the WebNLG and Cleaned E2E partitions combined, despite having smaller number of $\langle$ triple-set, sentence $\rangle$ pairs. This contributes significantly to the domain diversity of DART. In addition, we can see that DART exhibits a great deal of topical variety in terms of number of unique triples and vocabulary size.

\subsection{Dataset Split}

For WebNLG 2017 and Cleaned E2E, we use their original data splits. For our annotation on WikiTableQuestions and WikiSQL, random splitting will make train, dev, and test splits contain similar tables and similar /triple-set, sentence) examples. Therefore, to increase the generalization challenge, we compare the table title and the table header to find similar tables, and make sure the model is evaluated on test split tables that are least similar to those used for training. We first sample some tables as a seed test set, and then compute Jaccard similarity ${ }^{6}$ with remaining tables based on the titles and the headers. If a table has a Jaccard similarity greater than 0.5 with any of the tables in the test set, we add it into the test set. A similar process is repeated to create the dev set, and the remaining tables form the training set. This results in $62,659 / 6,980 / 12,552$ sentences in the train/dev/test sets, respectively.

\section{Experimental Results}

We conduct experiments on DART and the WebNLG 2017 dataset, with an ablation study on

\footnotetext{
${ }^{6}$ https://en.wikipedia.org/wiki/ Jaccard_index
} 
WebNLG to show the benefits of using DART for data augmentation.

\subsection{Models}

We investigate several state-of-the-art Data-to-Text generation models. We report results of the following models on DART-testset: (1) BidirectionalLSTM with attention, for which we use 2-layer bi-LSTM for encoder, with 300 dimensional word embeddings (without using pretrained word vectors), 512 hidden units and 0.3 dropout rate for the decoder. (2) Transformer (Vaswani et al., 2017), previously used by Castro Ferreira et al. (2019) on the WebNLG dataset. The input is formed by linearizing the unordered triple set. (3) BART (Lewis et al., 2020), for which we report results of both BART-base and BART-large. (4) T5 (Raffel et al., 2020): we add the same prefix "translate Graph to English:" to the input, as it is used in Ribeiro et al. (2020). We report results of T5-small, T5-base and T5-large models. For both BART and T5 models, we use implementations of Ribeiro et al. (2020), with same hyperparameter setting.

\subsection{Evaluation Metrics}

We use a variety of automatic metrics and human evaluation (Section 4) to evaluate the quality of the generated text. We report BLEU, METEOR, and TER which are used in the official WebNLG challenge. However, these measures have limitations in considering the semantic meanings of words or phrases (Novikova et al., 2017a), therefore we also report MoverScore (Zhao et al., 2019), BERTScore (Zhang et al., 2020), and BLEURT (Sellam et al., 2020) that incorporate semantics rather than surface forms using contextual embeddings. Furthermore, we include PARENT (Dhingra et al., 2019) which explicitly aligns n-grams from the reference and generated text to the data contents.

\subsection{Results}

DART Our experimental results on DART are summarized in Table 3. The T5-large model has the highest performance among all models with a BLEU score of 50.66. We attribute this to T5's generalization and transfer learning ability due to pretraining on multi-tasks. We can see that in general, pretrained models outperform others by a large margin, and increasing the model size seems to further boost the performance on DART. However, language models such as BART and T5 are pretrained by reconstructing text and, as a result, we found that their output on DART often contains hallucinated words (Parikh et al., 2020; Harkous et al., 2020; Reiter, 2020), as shown in Figure 11. In addition, while the pretrained model shows better text generation quality due to its generalization ability from pretraining, it does not fully capture the hierarchical ontology nature of the triple sets in their linearized input, therefore making DART more challenging. We suspect that models that are better at exploiting the ontology structure preserved in the input tripleset will achieve better performance on DART.

WebNLG Furthermore, we investigate if DART can improve pretrained models' performance on other Data-to-Text generation tasks. To this end, we finetune the baseline transformer model, BART-[base, large] and T5-[small, base, large] on the WebNLG 2017 dataset, and augment the training by adding instances in the DART training set. The experimental results can be found in Table 4 . We report performances of some competitive models that are not pretrained, as well as the state-of-the-art performances of pretrained models on the WebNLG 2017 dataset by Ribeiro et al. (2020). On the bottom panel, we include results of experiments augmented with DART instances whose triplesets are generated with table ontology annotation, paired with human written sentences. We are able to achieve new state-of-the-art results on all WebNLG 2017 test set splits (seen, unseen and all) by finetuning T5-large on DART. We observe that using DART for data augmentation consistently improves the performance across all models, including the baseline transformer model that is not pretrained. Furthermore, we observe that more improvement is shown on unseen split of the test set, due to DART's open-domain nature. See Figure 12 of the Appendix for example model outputs aligned with their human references.

\subsection{Ablation Study}

We also conduct an ablation study on the WebNLG dataset to investigate what part of DART contributes most to improving the Data-to-Text tasks in general. We report results of the study in Table 6 of the Appendix. We divide DART into 4 partitions, where declarative sentence (auto-generated) partition and human annotated sentence partition contain instances whose triplesets are extracted from Wikipedia tables based on ontology. E2E partition contains instances converted from the E2E 


\begin{tabular}{lccccccc}
\hline & BLEU $\uparrow$ & METEOR $\uparrow$ & TER $\downarrow$ & MoverScore $\uparrow$ & BERTScore $(F 1) \uparrow$ & BLEURT $\uparrow$ & PARENT $\uparrow$ \\
\hline LSTM with Attention & 29.66 & 0.27 & 0.63 & 0.31 & 0.90 & -0.13 & 0.35 \\
End-to-End Transformer & 27.24 & 0.25 & 0.65 & 0.25 & 0.89 & -0.29 & 0.28 \\
BART-base & 47.11 & 0.38 & 0.46 & 0.51 & 0.95 & 0.37 & 0.55 \\
BART-large & 48.56 & 0.39 & 0.45 & 0.52 & 0.95 & 0.41 & 0.57 \\
T5-small & 47.69 & 0.39 & 0.46 & 0.52 & 0.95 & 0.40 & 0.56 \\
T5-base & 49.21 & 0.40 & 0.44 & 0.53 & 0.95 & 0.43 & 0.57 \\
T5-large & $\mathbf{5 0 . 6 6}$ & $\mathbf{0 . 4 0}$ & $\mathbf{0 . 4 3}$ & $\mathbf{0 . 5 4}$ & $\mathbf{0 . 9 5}$ & $\mathbf{0 . 4 4}$ & $\mathbf{0 . 5 8}$ \\
\hline
\end{tabular}

Table 3: Model results on the test set of DART $\uparrow$ : Higher is better. $\downarrow$ : Lower is better.

\begin{tabular}{|c|c|c|c|c|c|c|c|c|c|}
\hline & \multicolumn{3}{|c|}{$\mathrm{BLEU} \uparrow$} & \multicolumn{3}{|c|}{ METEOR $\uparrow$} & \multicolumn{3}{|c|}{ TER $\downarrow$} \\
\hline & SEEN & UNSEEN & ALL & SEEN & UNSEEN & ALL & SEEN & UNSEEN & ALL \\
\hline Pipeline Transformer ${ }^{\dagger}$ (Castro Ferreira et al., 2019) & 56.28 & 23.04 & 42.41 & 0.42 & 0.21 & 0.32 & 0.39 & 0.63 & 0.50 \\
\hline Pipeline $\mathrm{GRU}^{\dagger}$ (Castro Ferreira et al., 2019) & 56.09 & 25.12 & 42.73 & 0.42 & 0.22 & 0.33 & 0.39 & 0.64 & 0.51 \\
\hline MELBOURNE (Gardent et al., 2017) & 54.52 & 33.27 & 45.13 & 0.41 & 0.33 & 0.37 & 0.40 & 0.55 & 0.47 \\
\hline BestPlan $^{\dagger}$ (Moryossef et al., 2019) & 53.30 & 34.41 & 47.24 & 0.44 & 0.34 & 0.39 & 0.47 & 0.56 & 0.51 \\
\hline DualEnc (Zhao et al., 2020) & 63.45 & 36.73 & 51.42 & 0.46 & 0.37 & 0.41 & 0.34 & 0.55 & 0.44 \\
\hline PlanEnc (Zhao et al., 2020) & 64.42 & 38.23 & 52.78 & 0.45 & 0.37 & 0.41 & 0.33 & 0.53 & 0.42 \\
\hline \multicolumn{10}{|l|}{ Ribeiro et al. (2020) } \\
\hline BART-base ${ }^{\ddagger}$ & 63.02 & 41.74 & 53.36 & 0.45 & 0.35 & 0.40 & 0.33 & 0.52 & 0.42 \\
\hline BART-large $\ddagger$ & 63.71 & 44.17 & 54.95 & 0.46 & 0.39 & 0.42 & 0.33 & 0.51 & 0.41 \\
\hline T5-small $\ddagger$ & 65.30 & 45.58 & 56.57 & 0.46 & 0.39 & 0.43 & 0.32 & 0.49 & 0.40 \\
\hline T5-base ${ }^{\ddagger}$ & 64.89 & 52.86 & 59.44 & 0.46 & 0.42 & 0.44 & 0.33 & 0.42 & 0.37 \\
\hline T5-large $\ddagger$ & 64.89 & 54.01 & 59.95 & 0.46 & 0.43 & 0.44 & 0.34 & 0.41 & 0.37 \\
\hline \multicolumn{10}{|l|}{ + DART } \\
\hline BART-base & 62.36 & 46.21 & 55.14 & 0.44 & 0.37 & 0.41 & 0.34 & 0.45 & 0.39 \\
\hline BART-large & 64.51 & 50.20 & 58.06 & 0.46 & 0.40 & 0.43 & 0.32 & 0.44 & 0.38 \\
\hline T5-small & 65.05 & 47.81 & 57.32 & 0.46 & 0.40 & 0.43 & 0.33 & 0.46 & 0.39 \\
\hline T5-base & 65.42 & 50.71 & 58.80 & 0.46 & 0.41 & 0.44 & 0.32 & 0.43 & 0.37 \\
\hline T5-large & 65.82 & 56.01 & 61.44 & 0.46 & 0.43 & 0.45 & 0.32 & 0.38 & 0.35 \\
\hline
\end{tabular}

Table 4: The WebNLG 2017 results on the test set. ${ }^{\dagger}$ : We report results from Zhao et al. (2020) who use the evaluation scripts that are strictly the same as the official challenge. ${ }^{\ddagger}$ : We report results calculated with the model outputs on the WebNLG 2017 testset released by Ribeiro et al. (2020).

\begin{tabular}{cccccc}
\hline Tripleset source & Sentence source & \% fluent & \% faithful & $\begin{array}{c}\text { \% (fluent+ } \\
\text { mostly fluent) }\end{array}$ & $\begin{array}{c}\text { \% (faithful+ } \\
\text { mostly faithful) }\end{array}$ \\
\hline \multirow{3}{*}{ WikiTableQuestions $(\$ \mathbf{2 . 1})$} & human-written reference & $75 \%$ & $81 \%$ & $96 \%$ & $99 \%$ \\
& BART-base & $74 \%$ & $57 \%$ & $93 \%$ & $84 \%$ \\
& T5-base & $72 \%$ & $54 \%$ & $94 \%$ & $76 \%$ \\
\hline \multirow{2}{*}{ WikiSQL (\$ 2.2) } & auto-generated reference & $59 \%$ & $56 \%$ & $87 \%$ & $88 \%$ \\
& BART-base & $66 \%$ & $51 \%$ & $92 \%$ & $83 \%$ \\
& T5-base & $75 \%$ & $65 \%$ & $97 \%$ & $90 \%$ \\
\hline
\end{tabular}

Table 5: Human evaluation over references and model outputs.

dataset, and WebNLG partition keeps the original data format. In general, we observe that adding DART instances that contain human written sentences brings most improvement, especially on unseen split. While adding E2E partition boosts the scores on seen test split and deteriorates the performance on unseen test split. This trend is consistent across all models. Comparing results of declarative sentence partition and human written sentence partition, we see that for most of the models, DART instances with human written sentences have better quality as it brings more improvement to the task.

\section{Human Evaluation}

In Table 5, we perform human evaluation on DART based on two criteria: (1) fluency if a sentence is natural and grammatical, and (2) semantic faithfulness if a sentence is supported by the input triples. We defined three levels of fluency: fluent, mostly fluent, and not fluent, and the same for semantic faithfulness. We ask 5 internal annotators to evaluate on 100 triplesets sampled from declarative sentence partition and another 100 triplesets sampled from human written sentence partition. Each 
tripleset is paired with 3 sentences, one of them is the reference sentence, and the other two are outputs of BART-base and T5-base models.

The results in Table 5 attest to the high quality of our annotations since the human written references achieve highest fluency and faithfulness comparing to outputs of two strong baseline models. The evaluation on faithfulness also demonstrates that there is a considerable gap between the DART reference and the outputs of the state-of-the-art pretrained model, showing that there is a large room for improvement. We also noticed that the auto-generated declarative sentences are not as fluent or faithful as the model outputs because they are generated with a rule-based system. However, we decided to release this partition, along with other partitions of DART because it demonstrates an economic way to obtain large amounts of DART instances and it also shows benefits for generalization due to the diverse topics it contains.

\section{Related Work}

Data-to-Text Data-to-Text generation aims to produce natural language output from structured input. Applications include generating sports commentaries (Chen and Mooney, 2008; Wiseman et al., 2017), weather forecasts (Liang et al., 2009; Konstas and Lapata, 2012), biographical texts (Lebret et al., 2016; Liu et al., 2018), knowledge-base descriptions (Gardent et al., 2017), dialogue response generation (Wen et al., 2015, 2016), and commonsense reasoning (Lin et al., 2020). Yet, most existing datasets are restricted to specific domains and applications. In contrast, a major source of DART is from Wikipedia tables covering various domains and topics.

Representation of Data The input of the Datato-Text datasets take different formats, including slot-value pairs, Abstract Meaning Representation (AMR) (Song et al., 2017; Ribeiro et al., 2019), Minimal Recursion Semantics (MRS) (Hajdik et al., 2019), Resource Description Framework (RDF triples) (Gardent et al., 2017), and logic forms (Chen et al., 2020b). There are also studies of converting tabular data to RDF triples in the Semantic Web community (Kellogg et al., 2015). Recently, some open-domain table-to-text datasets have been proposed including WikiTableText (Bao et al., 2018), LogicNLP (Chen et al., 2020a), and ToTTo (Parikh et al., 2020), whose inputs are rows or entire tables. In ToTTo, highlighted cells are also provided as input, and the authors found using only highlighted cells with flat row and column headers led to higher performance than using the entire table.

In contrast, DART is constructed by first annotating the tree-structured table ontology that encodes the semantic dependencies among table headers, and we could flexibly incorporate additional contexts such as the table title to the ontology tree. We then use an automatic procedure to extract connected components from the tree to form the input of a DART instance. Our annotation framework not only provides a flexible way of incorporating any contexts to the representation of tables, but also encodes hierarchical relationships among table headers and contexts, ensuring the extracted triples are logically consistent and can be described in text without loss of information.

Model Traditional Data-to-Text models break the generation progress into different stages such as signal analysis, data interpretation, document planning, microplanning, and realization (Reiter and Dale, 2000; Reiter, 2007). Recently, neural encoder-decoder models based on attention and copy mechanisms have shown promising results (Gehrmann et al., 2018; Puduppully et al., 2018, 2019; Castro Ferreira et al., 2019). Furthermore, recent progress on pretrained models such as GPT2 (Radford et al., 2018), BART (Lewis et al., 2020) and T5 (Raffel et al., 2020) has shown effective results for text generation tasks on machine translation, summarization, and conversation response generation. Chen et al. (2020c); Peng et al. (2020); Kale (2020) also finetune pretrained models on Data-to-Text tasks.

\section{Conclusion}

In this paper, we introduce DART, an open-domain corpus for structured data record to text generation. DART's ontology-preserving representation of data inputs differentiates itself from other open-domain Data-to-Text corpora. We found that DART introduces new challenges to several state-of-the-art Data-to-Text models due to its open-domain nature and its ontology structure of the semantic triple input. Furthermore, we found that using it for data augmentation improves other Data-to-Text tasks. For future work, we will explore more controlled, high-fidelity generation that better incorporates the ontology hierarchy of data. 


\section{Ethics Statement}

Our dataset is constructed by accumulating and processing resources from various existing datasets that are open to the public. In addition, we collect annotations on structure of tabular data and human written sentences that describe data records.

The existing resources that we utilize mainly consist of (1) tabular data from Wikipedia, (2) information of restaurants presented with dialogueact meaning representation and its textual description (E2E), and (3) information of various entities and their relationship that are in 15 different categories of DBPedia, which is a knowledge base built on contents created in various Wikimedia projects (WebNLG). It is possible that there are biases in these resources, either in the tabular data or the textual description written by humans.

For additional annotations we collected, we have two groups of annotators participating: internal annotators who are the authors of this work, and external annotators recruited from the Amazon Mechanical Turk platform. On MTurk, we use a pay rate of $\$ 15$ per hour approximately based on our estimation of the time it takes to complete our annotation tasks. In total, it took 125 hours to complete all tasks on the Amazon Mechanical Turk platform. There are three annotation tasks: (1) Annotators are asked to specify ontological structure of the table by indicating relationship between table column headers, (2) Annotators are asked to write descriptions that are fluent and semantically faithful to the data records presented to them, and (3) Annotators are asked to evaluate sentences that are either references or model generated outputs. We acknowledge that it is also possible to have biases in the sentences written by the annotators, or in the data records that are presented to them.

We conducted experiments on our own dataset and the WebNLG dataset using BART and T5, two large-scale pretrained models. Both models are trained on large amounts of textual data such as news, books, and web text, which may contain any kinds of biases. As a result, it is possible to insert those biases into the models.

In total, we conducted 43 experiments: 7 on DART and 36 for our ablation study on the WebNLG dataset. We use a single NVIDIA V100 GPU for all experiments and each experiment took from 5 to 40 hours depending on the model size.

\section{Acknowledgement}

The authors would like to thank the anonymous reviewers for their discussion and feedback.

\section{References}

Junwei Bao, Duyu Tang, Nan Duan, Zhao Yan, Yuanhua Lv, Ming Zhou, and Tiejun Zhao. 2018. Tableto-text: Describing table region with natural language. In $A A A I$.

Thiago Castro Ferreira, Chris van der Lee, Emiel van Miltenburg, and Emiel Krahmer. 2019. Neural datato-text generation: A comparison between pipeline and end-to-end architectures. In EMNLP.

Alison J Cawsey, Bonnie L Webber, and Ray B Jones. 1997. Natural language generation in health care.

David L Chen and Raymond J Mooney. 2008. Learning to sportscast: a test of grounded language acquisition. In ICML.

Wenhu Chen, Jianshu Chen, Yu Su, Zhiyu Chen, and William Yang Wang. 2020a. Logical natural language generation from open-domain tables. In $A C L$.

Zhiyu Chen, Wenhu Chen, Hanwen Zha, Xiyou Zhou, Yunkai Zhang, Sairam Sundaresan, and William Yang Wang. 2020b. Logic2Text: Highfidelity natural language generation from logical forms. In Findings of EMNLP.

Zhiyu Chen, Harini Eavani, Wenhu Chen, Yinyin Liu, and William Yang Wang. 2020c. Few-shot nlg with pre-trained language model. In $A C L$.

Dorottya Demszky, Kelvin Guu, and Percy Liang. 2018. Transforming question answering datasets into natural language inference datasets. arXiv preprint arXiv:1809.02922.

Bhuwan Dhingra, Manaal Faruqui, Ankur Parikh, Ming-Wei Chang, Dipanjan Das, and William Cohen. 2019. Handling divergent reference texts when evaluating table-to-text generation. In $A C L$.

Ondřej Dušek, David M. Howcroft, and Verena Rieser. 2019. Semantic noise matters for neural natural language generation. In $I N L G$.

Hady Elsahar, Pavlos Vougiouklis, Arslen Remaci, Christophe Gravier, Jonathon Hare, Frederique Laforest, and Elena Simperl. 2018. T-REx: A large scale alignment of natural language with knowledge base triples. In LREC.

Angela Fan, Claire Gardent, Chloé Braud, and Antoine Bordes. 2019. Using local knowledge graph construction to scale Seq2Seq models to multidocument inputs. In EMNLP-IJCNLP.

Claire Gardent, Anastasia Shimorina, Shashi Narayan, and Laura Perez-Beltrachini. 2017. The WebNLG challenge: Generating text from RDF data. In INLG. 
Sebastian Gehrmann, Falcon Dai, Henry Elder, and Alexander Rush. 2018. End-to-end content and plan selection for data-to-text generation. In INLG.

Valerie Hajdik, Jan Buys, Michael Wayne Goodman, and Emily M Bender. 2019. Neural text generation from rich semantic representations. In NAACL.

Hamza Harkous, Isabel Groves, and Amir Saffari. 2020 Have your text and use it too! end-to-end neural data-to-text generation with semantic fidelity. arXiv preprint arXiv:2004.06577.

Mihir Kale. 2020. Text-to-text pre-training for data-totext tasks. arXiv preprint arXiv:2005.10433.

Gregg Kellogg, Ivan Herman, and Jeremy Tandy. 2015. Generating RDF from tabular data on the web. W3C recommendation, W3C. Https://www.w3.org/TR/2015/REC-csv2rdf$20151217 /$.

Ioannis Konstas and Mirella Lapata. 2012. Unsupervised concept-to-text generation with hypergraphs. In NAACL.

Rémi Lebret, David Grangier, and Michael Auli. 2016. Neural text generation from structured data with application to the biography domain. In EMNLP.

Mike Lewis, Yinhan Liu, Naman Goyal, Marjan Ghazvininejad, Abdelrahman Mohamed, Omer Levy, Ves Stoyanov, and Luke Zettlemoyer. 2020. BART: Denoising sequence-to-sequence pretraining for natural language generation, translation, and comprehension. In $A C L$.

Percy Liang, Michael I Jordan, and Dan Klein. 2009 Learning semantic correspondences with less supervision. In $A C L$.

Bill Yuchen Lin, Wangchunshu Zhou, Ming Shen, Pei Zhou, Chandra Bhagavatula, Yejin Choi, and Xiang Ren. 2020. CommonGen: A constrained text generation challenge for generative commonsense reasoning. In Findings of EMNLP.

Tianyu Liu, Kexiang Wang, Lei Sha, Baobao Chang, and Zhifang Sui. 2018. Table-to-text generation by structure-aware seq2seq learning. In $A A A I$.

Amit Moryossef, Yoav Goldberg, and Ido Dagan. 2019 Step-by-step: Separating planning from realization in neural data-to-text generation. In $N A A C L$.

Jekaterina Novikova, Ondřej Dušek, Amanda Cercas Curry, and Verena Rieser. 2017a. Why we need new evaluation metrics for nlg. In EMNLP.

Jekaterina Novikova, Ondrej Dusek, and Verena Rieser. 2017b. The E2E dataset: New challenges for end-toend generation. In SIGDIAL.

Ankur Parikh, Xuezhi Wang, Sebastian Gehrmann, Manaal Faruqui, Bhuwan Dhingra, Diyi Yang, and Dipanjan Das. 2020. ToTTo: A controlled table-totext generation dataset. In EMNLP.
Panupong Pasupat and Percy Liang. 2015. Compositional semantic parsing on semi-structured tables. In $A C L$.

Baolin Peng, Chenguang Zhu, Chunyuan Li, Xiujun $\mathrm{Li}$, Jinchao Li, Michael Zeng, and Jianfeng Gao. 2020. Few-shot natural language generation for task-oriented dialog. In arXiv.

Ratish Puduppully, Li Dong, and Mirella Lapata. 2018. Data-to-text generation with content selection and planning. In $A A A I$.

Ratish Puduppully, Li Dong, and Mirella Lapata. 2019. Data-to-text generation with entity modeling. In $A C L$.

Alec Radford, Karthik Narasimhan, Tim Salimans, and Ilya Sutskever. 2018. Improving language understanding by generative pre-training. Technical report, OpenAI.

Colin Raffel, Noam Shazeer, Adam Roberts, Katherine Lee, Sharan Narang, Michael Matena, Yanqi Zhou, Wei Li, and Peter J. Liu. 2020. Exploring the limits of transfer learning with a unified text-to-text transformer. Journal of Machine Learning Research, 21(140):1-67.

Ehud Reiter. 2007. An architecture for data-to-text systems. In Proceedings of the Eleventh European Workshop on Natural Language Generation (ENLG 07).

Ehud Reiter. 2020. Openai gpt system: What does it do? Technical report, Arria.

Ehud Reiter and Robert Dale. 2000. Building natural language generation systems. Cambridge university press.

Leonardo F. R. Ribeiro, Claire Gardent, and Iryna Gurevych. 2019. Enhancing AMR-to-text generation with dual graph representations. In EMNLP.

Leonardo F. R. Ribeiro, Martin Schmitt, Hinrich Schütze, and Iryna Gurevych. 2020. Investigating pretrained language models for graph-to-text generation. arXiv.

Thibault Sellam, Dipanjan Das, and Ankur P Parikh. 2020. BLEURT: Learning robust metrics for text generation. In $A C L$.

Linfeng Song, Xiaochang Peng, Yue Zhang, Zhiguo Wang, and Daniel Gildea. 2017. Amr-to-text generation with synchronous node replacement grammar. In $A C L$

Ashish Vaswani, Noam Shazeer, Niki Parmar, Jakob Uszkoreit, Llion Jones, Aidan N Gomez, Łukasz Kaiser, and Illia Polosukhin. 2017. Attention is all you need. In NeurIPS. 
Pavlos Vougiouklis, Hady ElSahar, Lucie-Aimée Kaffee, Christophe Gravier, Frédérique Laforest, Jonathon S. Hare, and Elena Simperl. 2018. Neural wikipedian: Generating textual summaries from knowledge base triples. Journal of Web Semantics, 52-53:1 - 15 .

Tsung-Hsien Wen, Milica Gašić, Nikola Mrkšić, Lina M. Rojas-Barahona, Pei-Hao Su, Stefan Ultes, David Vandyke, and Steve Young. 2016. Conditional generation and snapshot learning in neural dialogue systems. In EMNLP.

Tsung-Hsien Wen, Milica Gašić, Nikola Mrkšić, PeiHao Su, David Vandyke, and Steve Young. 2015. Semantically conditioned LSTM-based natural language generation for spoken dialogue systems. In EMNLP.

Sam Wiseman, Stuart Shieber, and Alexander Rush 2017. Challenges in data-to-document generation. In EMNLP.

Tianyi Zhang, Varsha Kishore, Felix Wu, Kilian Q Weinberger, and Yoav Artzi. 2020. BERTScore: Evaluating text generation with BERT. In ICLR.

Chao Zhao, Marilyn Walker, and Snigdha Chaturvedi. 2020. Bridging the structural gap between encoding and decoding for data-to-text generation. In $A C L$.

Wei Zhao, Maxime Peyrard, Fei Liu, Yang Gao, Christian M Meyer, and Steffen Eger. 2019. MoverScore: Text generation evaluating with contextualized embeddings and earth mover distance. In EMNLP.

Victor Zhong, Caiming Xiong, and Richard Socher. 2017. Seq2sql: Generating structured queries from natural language using reinforcement learning. CoRR, abs/1709.00103. 


\section{Appendix}

The Appendix contains the following contents:

- Results of the ablation study on WebNLG 2017 testset.

- Statistics of the table ontology annotations.

- Examples of tables that help illustrate DART's annotation procedure.

- Examples of model outputs.

\begin{tabular}{|c|c|c|c|c|c|c|c|c|c|c|}
\hline \multirow{2}{*}{ Model } & \multirow{2}{*}{ Experiment } & \multicolumn{3}{|c|}{ BLEU $\uparrow$} & \multicolumn{3}{|c|}{ METEOR $\uparrow$} & \multicolumn{3}{|c|}{ TER $\downarrow$} \\
\hline & & SEEN & UNSEEN & ALL & SEEN & UNSEEN & ALL & SEEN & UNSEEN & ALL \\
\hline \multirow{6}{*}{$\begin{array}{c}\text { Baseline } \\
\text { Transformer }\end{array}$} & [1] webnlg & 49.81 & 5.51 & 31.81 & 0.39 & 0.09 & 0.24 & 0.47 & 0.86 & 0.64 \\
\hline & [2] webnlg+dart_decl_sents & 52.31 & 8.96 & 39.98 & 0.40 & 0.07 & 0.25 & 0.45 & 0.79 & 0.60 \\
\hline & [3] webnlg+dart_human_annotated & 53.68 & 7.02 & 36.36 & 0.40 & 0.09 & 0.26 & 0.43 & 0.79 & 0.59 \\
\hline & [4] webnlg+dart_ontology & 53.40 & 8.54 & 38.51 & 0.41 & 0.08 & 0.26 & 0.44 & 0.80 & 0.60 \\
\hline & [5] webnlg+dart_e2e & 51.76 & 5.92 & 32.36 & 0.40 & 0.09 & 0.25 & 0.45 & 0.86 & 0.63 \\
\hline & [6] webnlg+dart_full & 54.99 & 8.64 & 39.11 & 0.40 & 0.08 & 0.25 & 0.42 & 0.81 & 0.60 \\
\hline \multirow{6}{*}{ BART-base } & [1] webnlg & 63.02 & 41.74 & 53.36 & 0.45 & 0.35 & 0.40 & 0.33 & 0.52 & 0.42 \\
\hline & [2] webnlg+dart_decl_sents & 62.71 & 42.51 & 53.64 & 0.45 & 0.36 & 0.40 & 0.34 & 0.51 & 0.41 \\
\hline & [3] webnlg+dart_human_annotated & 62.36 & 46.21 & 55.14 & 0.44 & 0.37 & 0.41 & 0.34 & 0.45 & 0.39 \\
\hline & [4] webnlg+dart_ontology & 62.62 & 46.74 & 55.54 & 0.44 & 0.38 & 0.41 & 0.34 & 0.45 & 0.39 \\
\hline & [5] webnlg+dart_e2e & 64.00 & 35.07 & 51.17 & 0.45 & 0.33 & 0.40 & 0.33 & 0.61 & 0.46 \\
\hline & [6] webnlg+dart_full & 63.66 & 45.48 & 55.52 & 0.45 & 0.37 & 0.41 & 0.33 & 0.47 & 0.40 \\
\hline \multirow{6}{*}{ BART-large } & [1] webnlg & 63.71 & 44.17 & 54.95 & 0.46 & 0.39 & 0.42 & 0.33 & 0.51 & 0.41 \\
\hline & [2] webnlg+dart_decl_sents & 65.18 & 46.79 & 56.79 & 0.46 & 0.39 & 0.42 & 0.32 & 0.48 & 0.40 \\
\hline & [3] webnlg+dart_human_annotated & 64.51 & $\mathbf{5 0 . 2 0}$ & 58.06 & 0.46 & 0.40 & 0.43 & 0.32 & 0.44 & 0.38 \\
\hline & [4] webnlg+dart_ontology & 64.19 & 49.62 & 57.65 & 0.46 & 0.39 & 0.43 & 0.33 & 0.45 & 0.38 \\
\hline & [5] webnlg+dart_e2e & 65.06 & 30.17 & 48.24 & 0.46 & 0.33 & 0.40 & 0.32 & 0.69 & 0.49 \\
\hline & [6] webnlg+dart_full & 65.24 & 47.96 & 57.44 & 0.46 & 0.39 & 0.43 & 0.32 & 0.46 & 0.39 \\
\hline \multirow{6}{*}{ T5-small } & [1] webnlg & 65.30 & 45.58 & 56.57 & 0.46 & 0.39 & 0.43 & 0.32 & 0.49 & 0.40 \\
\hline & [2] webnlg+dart_decl_sents & 64.18 & 46.61 & 56.27 & 0.46 & 0.39 & 0.43 & 0.33 & 0.48 & 0.40 \\
\hline & [3] webnlg+dart_human_annotated & 65.05 & 47.81 & 57.32 & 0.46 & 0.40 & 0.43 & 0.33 & 0.46 & 0.39 \\
\hline & [4] webnlg+dart_ontology & 65.17 & 47.49 & 57.24 & 0.46 & 0.39 & 0.43 & 0.32 & 0.47 & 0.39 \\
\hline & [5] webnlg+dart_e2e & 65.56 & 41.28 & 54.56 & 0.46 & 0.38 & 0.42 & 0.32 & 0.54 & 0.42 \\
\hline & [6] webnlg+dart_full & 64.70 & 47.56 & 57.01 & 0.46 & 0.39 & 0.43 & 0.33 & 0.47 & 0.39 \\
\hline \multirow{6}{*}{ T5-base } & [1] webnlg & 64.89 & 52.86 & 59.44 & 0.46 & 0.42 & 0.44 & 0.33 & 0.42 & 0.37 \\
\hline & [2] webnlg+dart_ & 65.44 & 50.80 & 58.81 & 0.46 & 0.41 & 0.44 & 0.32 & 0.43 & 0.37 \\
\hline & [3] webnlg+dart_human_annotated & 65.42 & 50.71 & 58.80 & 0.46 & 0.41 & 0.44 & 0.32 & 0.43 & 0.37 \\
\hline & [4] webnlg+dart_ontology & 65.17 & 51.49 & 59.04 & 0.46 & 0.41 & 0.44 & 0.33 & 0.43 & 0.37 \\
\hline & [5] webnlg+dart_e2e & 65.11 & 49.64 & 58.19 & 0.46 & 0.41 & 0.44 & 0.33 & 0.46 & 0.39 \\
\hline & [6] webnlg+dart_full & 65.99 & 51.68 & $\mathbf{5 9 . 5 0}$ & 0.46 & 0.42 & 0.44 & 0.32 & 0.43 & 0.37 \\
\hline \multirow{6}{*}{ T5-large } & [1] webnl & 64.89 & 54.01 & 59.95 & 0.46 & 0.43 & 0.44 & 0.34 & 0.41 & 0.37 \\
\hline & [2] webnlg+dart_decl_sents & 65.97 & 53.00 & 60.12 & 0.46 & 0.42 & 0.44 & 0.32 & 0.41 & 0.36 \\
\hline & [3] webnlg+dart_human_annotated & 65.82 & 56.01 & 61.44 & 0.46 & 0.43 & 0.45 & 0.32 & 0.38 & 0.35 \\
\hline & [4] webnlg+dart_ontology & 65.53 & 55.20 & 60.90 & 0.46 & 0.42 & 0.44 & 0.32 & 0.38 & 0.35 \\
\hline & [5] webnlg+dart_e2e & 66.27 & 54.13 & 60.76 & 0.46 & 0.43 & 0.45 & 0.32 & 0.41 & 0.36 \\
\hline & [6] webnlg+dart_full & 65.78 & 54.35 & 60.64 & 0.46 & 0.42 & 0.44 & 0.32 & 0.39 & 0.35 \\
\hline
\end{tabular}

Table 6: Results of ablation study on WebNLG 2017 testset. dart_decl_sents refers to DART partition that contains auto-generated declarative sentences mentioned in Section 2.2, dart_human_annotated refers to partition that contains human written sentences mentioned in Section 2.1, dart_ontology is the combination of dart_decl_sents and dart_human_annotated, and dart_e2e refers to DART partition containing instances extracted from E2E dataset, the process of which is mentioned in Section 2.3. Note that dart_full is the combination of dart_ontology and dart_e2e. 


\begin{tabular}{lcccc}
\hline & Tables & $\begin{array}{c}\text { Ontology depth } \\
\text { (min, med, max })\end{array}$ & $\begin{array}{c}\text { Nodes in ontology } \\
\text { (min, med, max) }\end{array}$ & $\begin{array}{c}\text { Branching factor } \\
\text { (mean) }\end{array}$ \\
\hline WikiTableQuestions & 2060 & $1,1,4$ & $2,6,25$ & 4.0 \\
WikiSQL & 3563 & $1,1,4$ & $3,7,25$ & 5.1 \\
\hline
\end{tabular}

Table 7: Properties of the ontology in the WikiTableQuestions and WikiSQL samples in DART. Branching factor refers to the average number of children across all non-leaf nodes in a table's ontology.

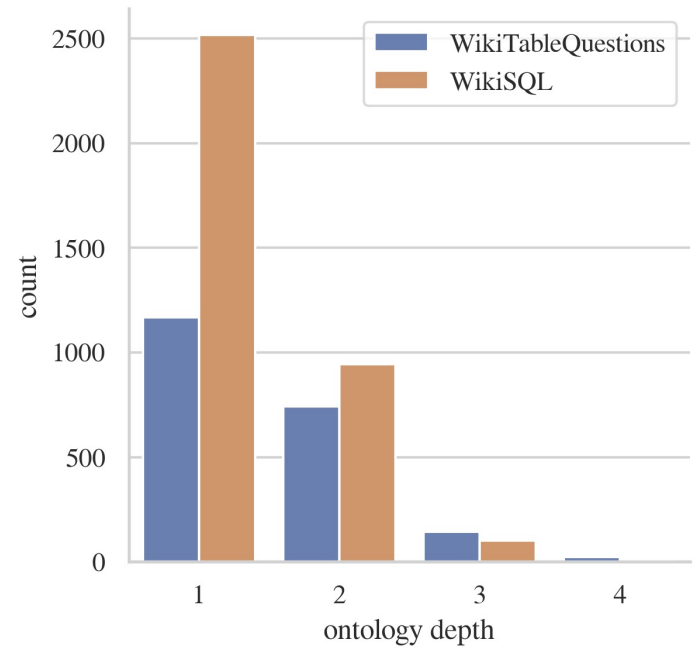

Figure 3: Distribution of column ontology depths in the WikiTableQuestions and WikiSQL samples in DART v1.1.1.

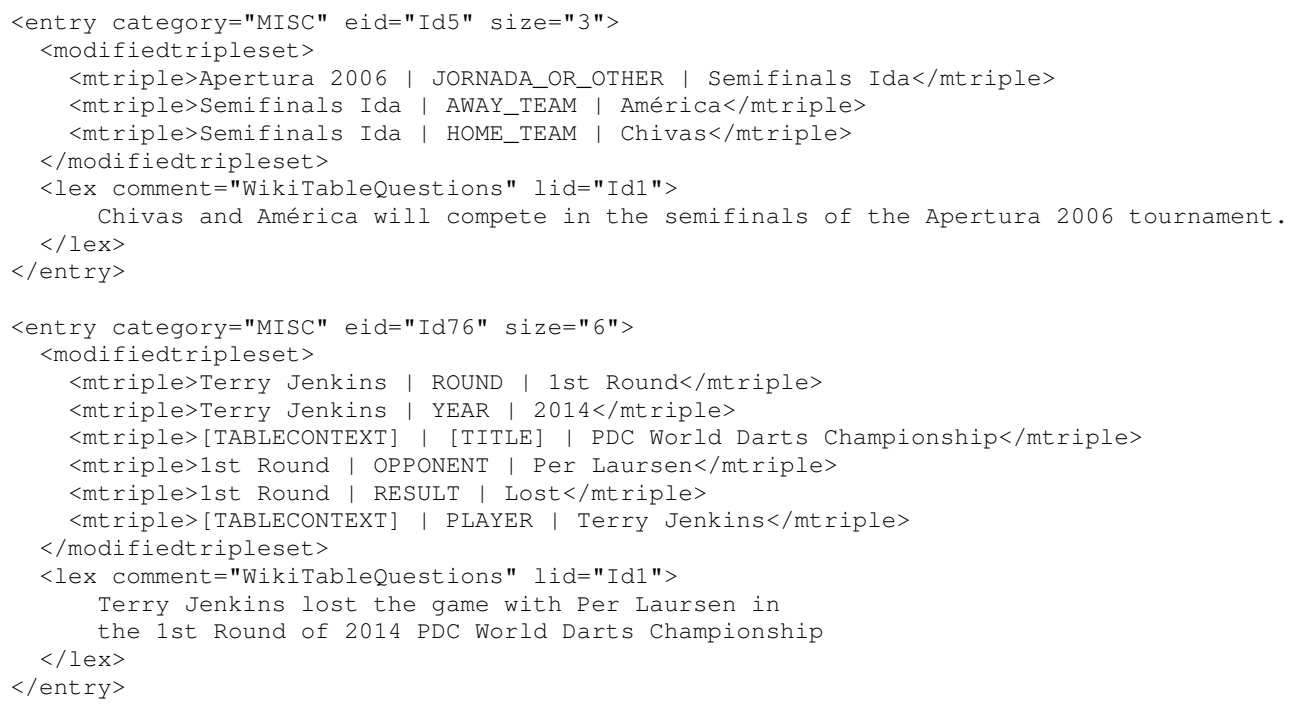

Figure 4: Examples of DART instance 


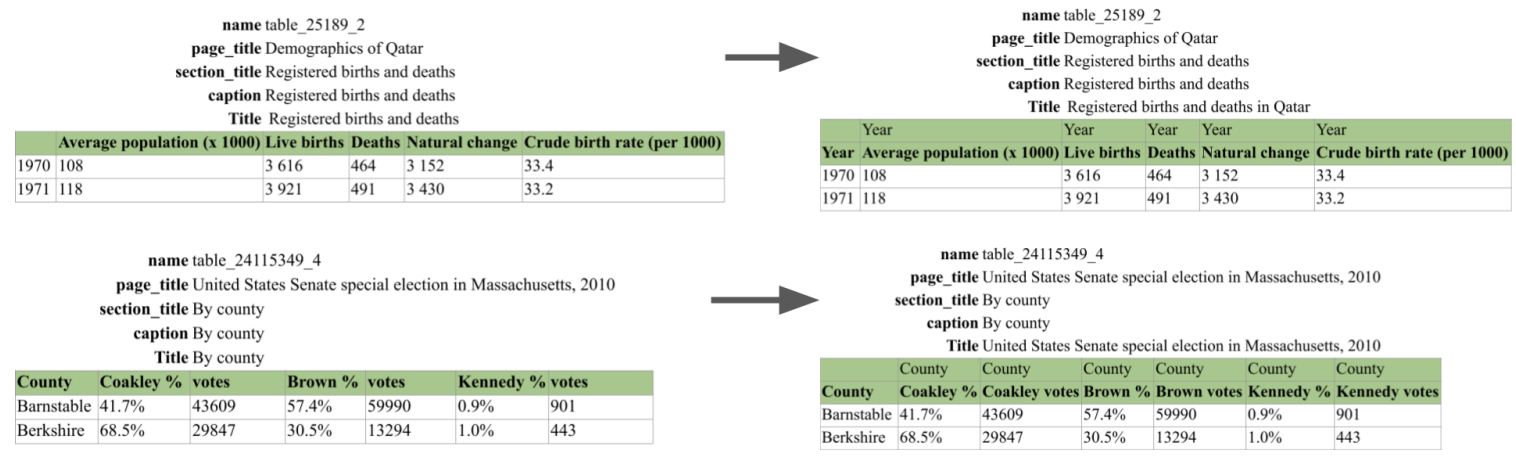

Figure 5: An example of the data cleaning. The top left table had a missing column name and the table title was not specific to the data; our internal annotators add the missing column name "Year" and linked the rest of the columns to the "Year" column. The bottom left table had repeat column names in the table; our internal annotators disambiguate the columns by making the column names more specific.

\begin{tabular}{|c|c|c|c|c|c|c|c|}
\hline [TITLE] & [TITLE] & [TITLE] & [TITLE] & [TITLE] & [TITLE] & [TITLE] & Class \\
\hline Year & Team & Co-Drivers & Car & Class & Laps & Pos. & Class Pos. \\
\hline 2001 & Team Den Blå Avis / Team Goh & John Nielsen \& Hiroki Katoh & Dome S101-Judd & LMP900 & 66 & DNF & DNF \\
\hline 2003 & RN Motorsport & John Nielsen \& Hayanari Shimoda & DBA4 03S-Zytek & LMP675 & 288 & $22 \mathrm{nd}$ & 2nd \\
\hline 2004 & Lister Racing & John Nielsen \& Jens Møller & Lister Storm LMP-Chevrolet & LMP1 & 279 & 24th & 9th \\
\hline 2006 & Zytek Engineering / Team Essex Invest & John Nielsen \& Philip Andersen & Zytek 06S & LMP1 & 269 & $\mathrm{NC}$ & $\mathrm{NC}$ \\
\hline 2007 & Aston Martin Racing Larbre & Christophe Bouchut \& Fabrizio Gollin & Aston Martin DBR9 & GT1 & 341 & 7 th & $3 \mathrm{rd}$ \\
\hline 2008 & Team Essex & John Nielsen \&Sascha Maassen & Porsche RS Spyder Evo & LMP2 & 347 & 12th & 2nd \\
\hline 2009 & Team Essex & Kristian Poulsen \& Emmanuel Collard & Porsche RS Spyder Evo & LMP2 & 357 & 10th & 1 st \\
\hline 2011 & Hope Racing & Steve Zacchia \& Jan Lammers & Oreca 01-Swiss HyTech & LMP1 & 115 & DNF & DNF \\
\hline
\end{tabular}

Figure 6: A WikiTableQuestions table that uses [TITLE] in the ontology.

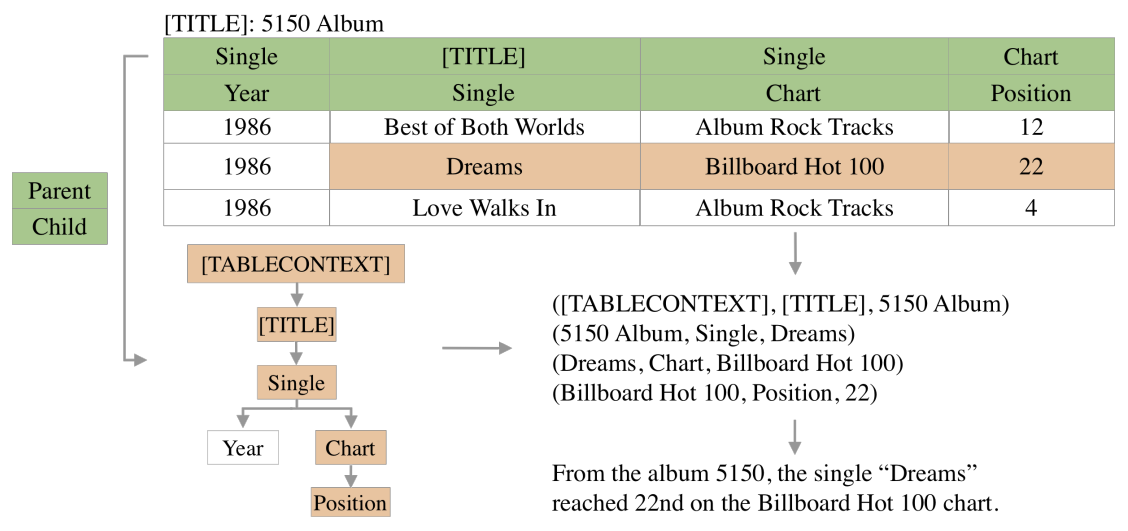

Figure 7: A manually annotated table from WikiTableQuestions with a sentence that uses the table title.

\begin{tabular}{|c|c|c|c|c|c|c|}
\hline Competition & Event & Competition & [TITLE] & Position & [TITLE] & \multirow{6}{*}{ The competitor won a silver medal at the 2000 World Junior Championships in Santiago, Chile } \\
\hline Year & Competition & Venue & Position & Event & Time & \\
\hline 2000 & World Junior Championships & Santiago, Chile & 2nd & $400 \mathrm{~m}$ hurdles & 50.52 & \\
\hline 2000 & World Junior Championships & Santiago, Chile & 4th & $4 \times 400 \mathrm{~m}$ relay & 3:07.66 & \\
\hline 2005 & World Championships & Helsinki, Finland & 17th (sf) & $400 \mathrm{~m}$ hurdles & 49.75 & \\
\hline 2005 & World Championships & Helsinki, Finland & 13th (h) & $4 \times 400 \mathrm{~m}$ relay & 3:04.64 & \\
\hline 2006 & Commonwealth Games & Melbourne, Australia & 7th & $400 \mathrm{~m}$ hurdles & 50.51 & They finished the $400 \mathrm{~m}$ hurdles 7 th in 50.51 . \\
\hline 2006 & African Championships & Bambous, Mauritius & 4th & $400 \mathrm{~m}$ hurdles & 50.96 & In Bambous, Peter De Villiers came in 4th in the African Championships. \\
\hline 2007 & All-Africa Games & Algiers, Algeria & 2nd & $400 \mathrm{~m}$ hurdles & 48.91 & \multirow{4}{*}{ The 2007 All-Africa Games held a $4 \times 400$ m relay. } \\
\hline 2007 & All-Africa Games & Algiers, Algeria & 8th & $4 \times 400 \mathrm{~m}$ relay & DNF & \\
\hline 2007 & World Championships & Osaka, Japan & 15th (sf) & $400 \mathrm{~m}$ hurdles & 49.37 & \\
\hline 2008 & Olympic Games & Beijing, China & 12th (sf) & $400 \mathrm{~m}$ hurdles & 49.44 & \\
\hline
\end{tabular}

Figure 8: A manually annotated table from WikiTableQuestions. Annotators created a table ontology, and they wrote sentences encapsulating the information in the orange cells for a given row. Whenever a sentence referenced the table title, that sentence was also highlighted green. 


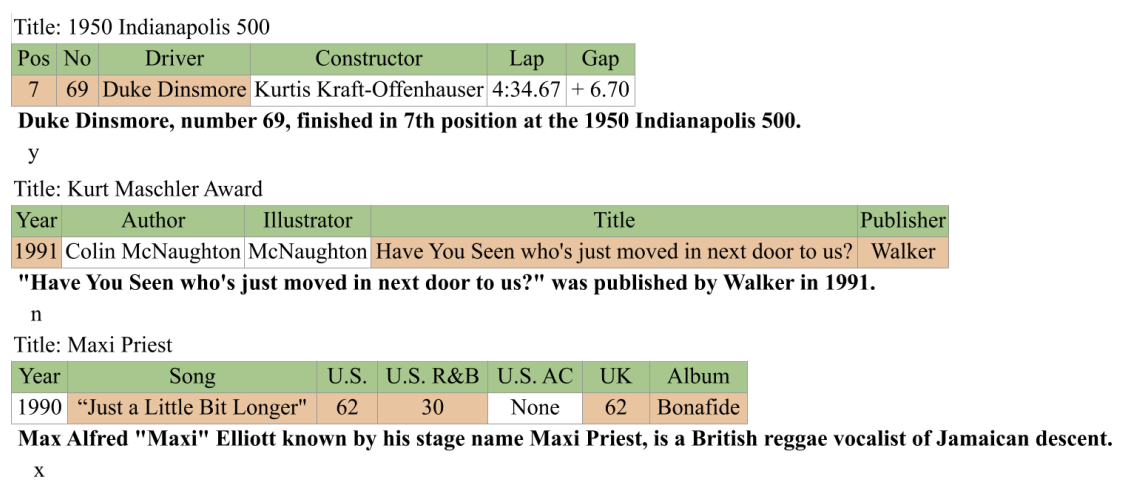

Figure 9: An example of collected MTurk-generated sentences for WikiTableQuestions. Internal annotators went through the generated sentences and checked for both sentence coherence and title usage. Below the generated sentences, ' $y$ ' meant the sentence references the table title, ' $n$ ' meant the sentence did not use the table title, ' $x$ ' meant the sentence was nonsensical.

\begin{tabular}{|c|c|c|c|c|c|c|}
\hline Year & Division & League & Regular Season & Playoffs & U.S. Open Cup & Avg. Attendance \\
\hline 2012 & 3 & usl pro & 6th & quarterfinals & quarterfinals & 1452 \\
\hline \multicolumn{7}{|c|}{$\begin{array}{l}\text { A team in the usl pro league has an average attendance of } 1452 . \\
n\end{array}$} \\
\hline Year & Division & League & Regular Season & Playoffs & U.S. Open Cup & Avg. Attendance \\
\hline 2009 & 3 & usl second division & $3 \mathrm{rd}$ & semifinals & quarterfinals & 1857 \\
\hline \multicolumn{7}{|c|}{$\begin{array}{l}\text { In 2009, the Harrisburg City Islanders got semifinals in Playoffs in league usl second division. } \\
\text { y }\end{array}$} \\
\hline Year & Division & League & Regular Season & Playoffs & Open Cup & \\
\hline 2008 & 4 & usl pdl & 4 th, great lakes & did not qualify & did not qualify & \\
\hline
\end{tabular}

Figure 10: Automatically generated declarative sentences from WikiSQL with human validation. Annotators went through the generated sentences and checked for both sentence coherence and title use. Below the generated sentences, ' $y$ ' meant the sentence references the table title, ' $n$ ' meant the sentence did not use the table title, ' $\mathrm{x}$ ' meant the sentence was nonsensical.

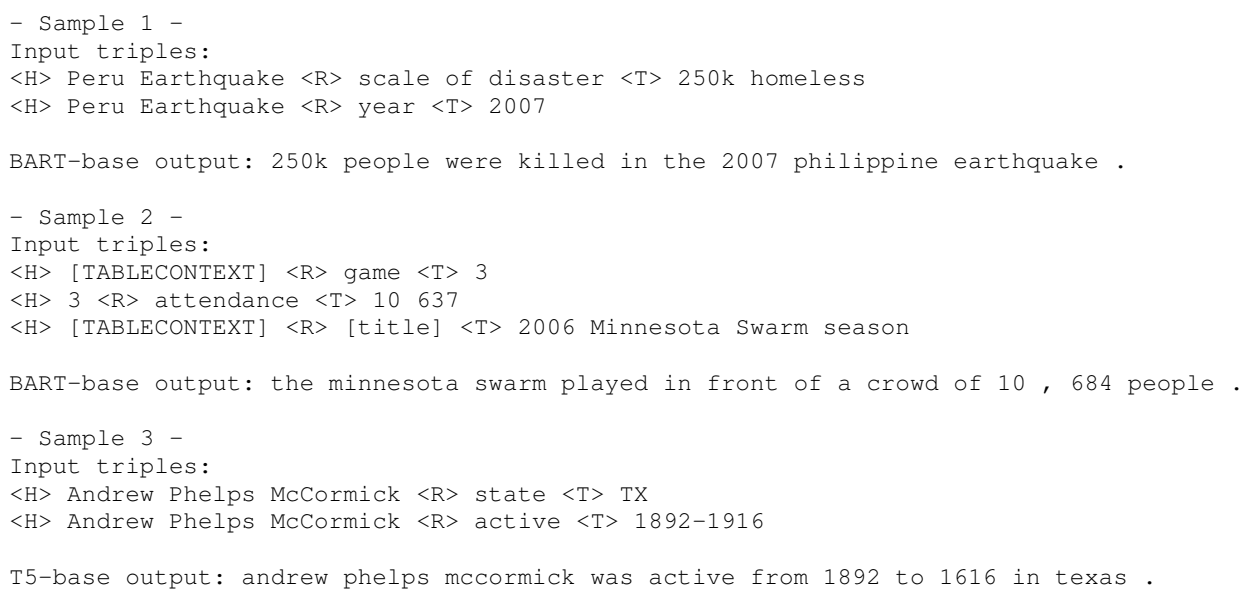

Figure 11: Examples of hallucinated outputs of pretrained models trained on DART 


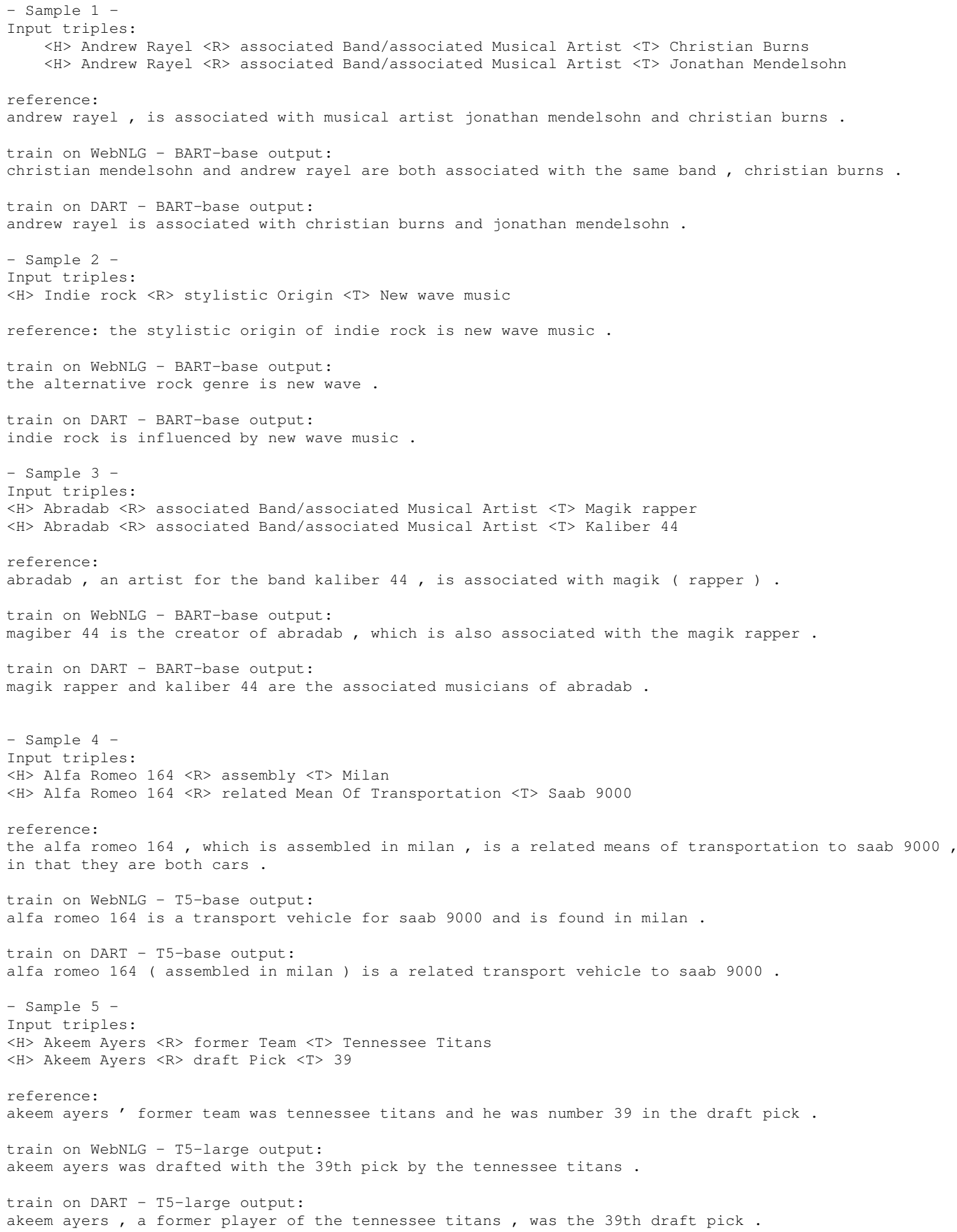

Figure 12: Examples of model outputs - with or without DART data augmentation 